\title{
Total perinatally related losses at Tygerberg Hospital - a comparison between 1986, 1993 and 2006
}

\author{
Quonita Talip, Gerhard Theron, Wilhelm Steyn, David Hall
}

Objective. To determine the leading causes of perinatal deaths and to evaluate any changes, with the inclusion of placental histology.

Method. At perinatal mortality meetings, primary and final causes of death were assigned for the period 1 July 2006 - 30 June 2007. All singleton babies born to women residing in the metropolitan area serviced by Tygerberg Hospital were included in the prospective descriptive study.

Results. The total number of singleton births was 10396 . The total of perinatally related losses (TPRL) rate was 26.2 per 1000 births. The leading primary obstetric causes of death were: infections $(47-17.3 \%)$, spontaneous preterm labour (PTL) $(41-15.1 \%)$, antepartum haemorrhage (APH) $(40$ $-14.7 \%)$, intra-uterine growth restriction (IUGR) $(40-14.7 \%)$, fetal abnormality $(31-11.4 \%)$, hypertensive disorders $(25$ $-9.2 \%)$, unexplained intra-uterine deaths (IUD) $(20-7.4 \%)$, intrapartum hypoxia $(12-4.4 \%)$ and maternal disease (9 $-3.3 \%$ ). A total of 162 placentas were sent for histology; 58 reports changed the primary cause of death.

Conclusion. The TPRL rate for singleton pregnancies was 26.2 per 1000 births for the study period. The TPRL rates in 1986 and 1993 were 36.7 and 30.5 per 1000 deliveries. Infection is now the leading primary cause of death, followed by spontaneous PTL, APH and IUGR. During the previous two study periods, APH was the leading primary cause of death, followed by spontaneous PTL. Unexplained IUDs ranked third in 1986, fourth in 1993 and seventh in this study because of the availability of placental histology. Placental histology reports changed $21.3 \%$ of the primary causes of death.

S Afr Med J 2010; 100: 250-253.
We aimed to identify the leading causes of perinatal deaths in the Tygerberg Hospital (TBH) drainage area in order to target care more effectively. The outcome of the audit was compared with two previous, similar studies, which ${ }^{1,2}$ highlighted trends over nearly 20 years regarding primary and final causes of deaths as well as perinatal mortality rates. Since the last published audit in 1993, placental histology has been included when determining the primary cause of death, ${ }^{2}$ and its effect on allocation of the primary cause of death was evaluated.

\section{Method}

A prospective descriptive study was made of the period 1 July 2006 - 30 June 2007. All singleton babies delivered from women residing in the metropolitan area directly serviced by TBH and the two midwife obstetrics units (MOUs) attached to TBH (namely, the Bishop Lavis and Elsies River MOUs) were included. The total perinatally related loss (TPRL) is the sum of all late abortions, stillbirths, early neonatal deaths and late neonatal deaths. ${ }^{1}$

A weekly perinatal mortality (PNM) meeting, attended by obstetricians, neonatologists, geneticists, pathologists, obstetric and paediatric registrars, medical officers and interns, is held in TBH's Department of Obstetrics and Gynaecology. At this meeting, each perinatal death involving a fetus weighing more

Department of Obstetrics and Gynaecology, Tygerberg Hospital and Stellenbosch University, Tygerberg, W Cape

Quonita Talip, MB ChB, FCOG (SA), MMed (O\&G)

Gerhard Theron, MB ChB, FCOG (SA), MMed (O\&G), BSc (Hons), MD

Wilhelm Steyn, MB ChB, FCOG ( SA), MMed (O\&G), MD (Stell)

David Hall, MB ChB, Dip Obst (SA), FCOG (SA), MMed (O\&G), MD

Corresponding author: Gerhard Theron (gbth@sun.ac.za) than $499 \mathrm{~g}$ is discussed and a primary and final cause of death is assigned. Avoidable factors are highlighted and recorded in the categories of patient, medical care and administrativerelated avoidable factors. ${ }^{3}$ Every intra-uterine death is examined by a geneticist for signs of congenital abnormality or infection of the baby or placenta.

According to a consensus protocol, certain placentas are sent for histological evaluation. Placental histology reports (when available) were presented at the meetings and, if the report changed the primary or final cause of death, it was recorded for the index study. The information collected at these meetings was recorded and transferred to a spreadsheet for the index study. Personal identification of patients was removed and the information collated and analysed.

The study was approved by the Committee for Human Research of the Faculty of Health Sciences of Stellenbosch University.

\section{Results}

The total number of singleton births was 10 396, including 111 pairs of twins. The TPRL for singleton pregnancies was 272 cases. There were 25 deaths among the twin pregnancies. The TPRL rate for singleton pregnancies was 26.2 per 1000 births. The PNM rate for infants $>499 \mathrm{~g}$ from only intra-uterine deaths (IUD) (209) and early neonatal deaths (55) was 25.3 per 1000 births. The leading primary obstetric causes of death in singleton pregnancies are listed in Table I. Treponemal infections were responsible for 18 of the 47 deaths where infection was the primary cause of death.

Losses according to weight category were highest $(41.5 \%)$ in the 500 - 999 g group; $16.5 \%$ losses occurred in the 1000 - $1499 \mathrm{~g}$ group, $13.2 \%$ in the $1500-1999$ g group, $11.0 \%$ in the 2000 2499 g group, and $17.6 \%$ in the $>2500$ g group (Table II). The 
Table I. The leading primary obstetric causes of perinatally related losses in singleton pregnancies

\begin{tabular}{|c|c|c|c|c|c|}
\hline Primary causes & Late abortions & Stillbirths & Early neonatal deaths & Late neonatal deaths & $N(\%)$ \\
\hline Infections & 18 & 22 & 7 & 0 & $47(17.3)$ \\
\hline Spontaneous PTL & 19 & 2 & 18 & 2 & $41(15.1)$ \\
\hline $\mathrm{APH}$ & 11 & 25 & 4 & 0 & $40(14.7)$ \\
\hline IUGR & 12 & 27 & 1 & 0 & $40(14.7)$ \\
\hline Fetal abnormality & 6 & 13 & 8 & 4 & $31(11.4)$ \\
\hline Hypertensive disorders & 11 & 4 & 9 & 1 & $25(9.2)$ \\
\hline Unexplained IUD & 6 & 14 & 0 & 0 & $20(7.4)$ \\
\hline Intrapartum hypoxia & 0 & 7 & 4 & 1 & $12(4.4)$ \\
\hline Maternal disease & 3 & 5 & 1 & 0 & $9(3.3)$ \\
\hline Other & 0 & 1 & 2 & 1 & $4(1.5)$ \\
\hline Birth trauma & 0 & 3 & 0 & 0 & $3(1.1)$ \\
\hline Total & 86 & 123 & 54 & 9 & 272 \\
\hline
\end{tabular}

Table II. Primary obstetric causes of perinatally related losses per weight category

\begin{tabular}{|c|c|c|c|c|c|c|}
\hline Primary causes & $500-999 g$ & $1000-1499 \mathrm{~g}$ & $1500-1999 \mathrm{~g}$ & $2000-2499 g$ & $>2500 \mathrm{~g}$ & $N(\%)$ \\
\hline Infections & 20 & 7 & 7 & 3 & 10 & $47(17.3)$ \\
\hline Spontaneous PTL & 32 & 4 & 3 & 2 & 0 & $41(15.1)$ \\
\hline $\mathrm{APH}$ & 14 & 9 & 6 & 9 & 2 & $40(14.7)$ \\
\hline IUGR & 12 & 10 & 6 & 3 & 9 & $40(14.7)$ \\
\hline Fetal abnormality & 8 & 7 & 5 & 6 & 5 & $31(11.4)$ \\
\hline Hypertensive disorders & 17 & 3 & 3 & 2 & 0 & $25(9.2)$ \\
\hline Unexplained IUD & 6 & 2 & 3 & 3 & 6 & $20(7.4)$ \\
\hline Intrapartum hypoxia & 0 & 0 & 1 & 1 & 10 & $12(4.4)$ \\
\hline Maternal disease & 4 & 1 & 1 & 0 & 3 & $9(3.3)$ \\
\hline Other & 0 & 2 & 1 & 1 & 0 & $4(1.4)$ \\
\hline Birth trauma & 0 & 0 & 0 & 0 & 3 & $3(1.1)$ \\
\hline Total (\%) & 113 (41.5) & $45(16.5)$ & $36(13.2)$ & $30(11.0)$ & 48 (17.6) & 272 \\
\hline
\end{tabular}

leading final causes of neonatal deaths are listed in Table III according to weight category. The highest neonatal losses were in the 500 - 999 g group, accounting for $42.2 \%$ of all losses.

A total of 162 placentas were sent for histological investigation. Of the subsequent reports, 58 contributed to changing the primary causes of death (for singleton pregnancies) and helped to assign the primary cause of death to another 46 cases which would otherwise have been unexplained (Table IV). With the benefit of placental histology reporting, 24 deaths in the latter group could be attributed to intra-uterine growth restriction (IUGR) and 18 to infection. The latter can be further subdivided into chorio-amnionitis (14), preterm labour (PTL) with chorio-amnionitis and intact membranes (3) and treponemal infection (1). The remaining 4 cases changed to abruptio placentae.

Placental histology reports helped to assign the correct diagnoses in 12 cases that otherwise would have had an incorrect primary cause of death assigned to them. Three cases of spontaneous PTL, 2 cases of chorio-amnionitis, and 1 case of hypertension all became IUGR (Table IV). One case thought to be hypoxia-related and 2 cases thought to be owing to cervical incompetence, became PTL with chorio-amnionitis and intact membranes. One case with a hypertensive disorder, and 2 cases thought to be maternal disease, became chorio-amnionitis. Placental histology reports changed $21.3 \%$ of the primary causes of death in the index study.

There were 240 avoidable factors, of which 209 were patient-, 19 medical care- and 12 administrative-related avoidable factors. The most common patient-related avoidable factors were: unbooked patients (73), patients booked late in pregnancy (32), patients who delayed seeking medical assistance (27), and patients with inappropriate responses to poor fetal movements (23). The most common avoidable factors related to medical care were: underestimation of fetal size (2) and lack of response by staff to patients with poor obstetric histories (2). The most common administrative-related avoidable factors were: unavailable operating theatres (5) and lack of transport between institutions (3).

\section{Discussion}

A unique opportunity existed at TBH to compare perinatal service over two decades. Comparing the new data with those from two previous studies allowed assessment of whether PNM rates and TPRL rates increased or decreased in the area serviced by TBH and the two attached MOUs. The methodology used in this study is the same as that of the previous two studies.

Discussing cases at PNM meetings provided the opportunity to explicate the causes of perinatal deaths, pregnancy complications, patient and health infrastructure interaction, and health workers' interventions, all of which were identified, ordered and classified.

TBH is a secondary/tertiary level hospital in the Western Cape, serving low to middle socio-economic population groups. Only patients residing in the direct TBH drainage area were included in the study, while patients referred from outlying areas were excluded. Most people residing in this area 


\section{Original Articles}

\begin{tabular}{|c|c|c|c|c|c|c|}
\hline $\begin{array}{l}\text { Final causes of } \\
\text { neonatal death }\end{array}$ & $500-999 g$ & $1000-1499 g$ & $1500-1999 g$ & $2000-2499 g$ & $>2500 \mathrm{~g}$ & $N(\%)$ \\
\hline Immaturity related & 22 & 3 & 1 & 0 & 0 & $26(40.6)$ \\
\hline Congenital abnormalities & 2 & 2 & 3 & 1 & 3 & $11(17.2)$ \\
\hline Intrapartum hypoxia & 0 & 1 & 1 & 3 & 5 & $10(15.6)$ \\
\hline Infection & 2 & 1 & 3 & 2 & 2 & $10(15.6)$ \\
\hline Unexplained causes & 0 & 0 & 1 & 3 & 1 & $5(7.8)$ \\
\hline Birth trauma & 1 & 0 & 0 & 0 & 0 & $1(1.6)$ \\
\hline Other & 0 & 1 & 0 & 0 & 0 & $1(1.6)$ \\
\hline Total & 27 & 8 & 9 & 9 & 11 & 64 \\
\hline
\end{tabular}

\section{Table IV. Primary cause of death that changed as a result of placental histology report}

\begin{tabular}{lll}
\hline$N$ & From & To \\
\hline 3 & Unknown (46) & PTL with chorio-amnionitis with intact membranes \\
14 & & Chorio-amnionitis \\
4 & & Abruptio placentae \\
1 & & Treponemal infection \\
24 & Spontaneous PTL & IUGR \\
3 & Chorio-amnionitis & IUGR \\
2 & Hypertension-related & IUGR \\
1 & Intrapartum hypoxia & IUGR \\
1 & Cervical incompetence & PTL with chorio-amnionitis with intact membranes \\
2 & Hypertension-related & PTL with chorio-amnionitis with intact membranes \\
1 & Maternal disease & Chorio-amnionitis \\
2 & & Chorio-amnionitis \\
\hline
\end{tabular}

have no access to private health care and, with rare exceptions, all the women intend to deliver within the health service. Therefore, the studies could be regarded as population-based.

The total number of deliveries at TBH steadily increased over the past 2 decades. ${ }^{1,2}$ IUD rates stayed steady between 1986 and 1993, and decreased between 1993 and 2007 by 27\% (Table V). The neonatal death (NND) rate reduced by $55.9 \%$ from 1986 to 1993 and showed a slight increase in 2007. The TPRL rate in 1986, 1993 and 2007 was respectively 36.7, 30.5 and 26.2 per 1000 deliveries. The TPRL rate for singleton pregnancies greater than $499 \mathrm{~g}$ over the past 2 decades decreased steadily by about $15 \%$, while the total number of deliveries increased by $31 \%$. This favourable trend is due to the decrease in the NND and IUD rates, and is reassuring, given the chronic staff shortages and limited resources. This decline has been sustained over two decades, and we can therefore conclude that a reasonable service is rendered to the population using public health services in the Tygerberg region.

During the interval between the first two studies (1986 and 1993), the NND rate decreased by about $60 \%{ }^{1,2}$ as a result of rapid advances in neonatal care, including the use of surfactant and improved neonatal intensive care. During 1993 and 2006, there was a reduction in the IUD rate. ${ }^{2}$ Specialists working in maternal-fetal medicine attributed part of the improvement to the introduction of umbilical artery Doppler investigation at TBH in 1995 to identify chronic placental insufficiency. Eligible patients included those at risk for placental insufficiency or a clinical suspicion of IUGR. ${ }^{4}$ However, clinical inspection and palpation to assess small-for-gestational-age (SGA) fetuses have a low sensitivity (44\%) and specificity $(88 \%) .{ }^{5}$ Serial ultrasound measurements remain one of the best methods
Table V. Intra-uterine deaths, neonatal deaths, total perinatal related losses and perinatal mortality rates for 1986, 1993 and 2007

\begin{tabular}{llll}
\hline Rate/1 000 births & 1986 & 1993 & 2007 \\
\hline Total number of singletons & 7923 & 8616 & 10396 \\
IUD & 27.4 & 26.5 & 20.0 \\
NND & 9.3 & 4.1 & 6.2 \\
TPRL & 36.7 & 30.5 & 26.2 \\
PNM & 33.9 & 29.2 & 25.3 \\
\hline
\end{tabular}

to monitor fetal growth. ${ }^{4}$ A combination of umbilical artery Doppler and ultrasound findings has a sensitivity of $81 \%$. The use of umbilical artery Doppler in high-risk pregnancies reduces the odds of perinatal death by $38 \%{ }^{7}$

In 1986, Pattinson et al. identified the leading primary obstetric causes of TPRL as being antepartum haemorrhage (APH), spontaneous PTL, unexplained IUD, infection and fetal abnormalities (Table VI). ${ }^{1}$ Prins et al. in 1993 then looked at changes in the mortality rate and found that the leading causes were $\mathrm{APH}$, spontaneous PTL, infection, unexplained IUD and fetal abnormalities. Infection, especially caused by syphilis, was increasing as a leading cause of death, even surpassing unexplained IUD. ${ }^{2}$

In our study, infection-related losses were found to be the highest primary obstetric cause of death, at $17.3 \%$. The availability of the placental histology service changed the diagnosis of the primary cause of death to chorio-amnionitis in 17 cases (14 of which were originally classified as unexplained) (Table IV), and to PTL with chorio-amnionitis and intact membranes in 6 cases. 
Table VI. Primary obstetric causes of deaths for the 3 study periods

\begin{tabular}{llll}
\hline & 1986 & 1993 & 2007 \\
\hline Spontaneous PTL & 24.1 & 20.5 & 15.1 \\
Infections & 9.6 & 14.1 & 17.3 \\
APH & 28.9 & 25.5 & 14.7 \\
IUGR & 4.8 & 1.5 & 14.7 \\
Hypertension & 5.2 & 11.4 & 9.2 \\
Fetal abnormality & 7.9 & 8 & 11.4 \\
Birth trauma & 1 & 0 & 1.1 \\
Intrapartum hypoxia & 3.8 & 2.7 & 4.4 \\
Maternal disease & 1.4 & 0.7 & 3.3 \\
Other & 1 & 2.2 & 1.4 \\
Unexplained IUD & 12.4 & 12.9 & 7.4 \\
\hline
\end{tabular}

The Confidential Enquiry into Maternal and Child Health (CEMACH) did not mention infection as a leading cause of death. ${ }^{8}$ Sub-clinical chorio-amnionitis has, however, been linked to IUDs and PTL. ${ }^{9}$ Acute chorio-amnionitis alone is rarely a cause of stillbirths, according to Yee Khong, except in the presence of overwhelming fetal sepsis. ${ }^{10}$ In our study, however, 14 (30.4\%) of the 46 IUDs of unexplained cause were changed to chorio-amnionitis. The study population is at high risk for infectious complications, often resulting in IUD. The high incidence of PTL in the study population combined with the placental pathology results suggest that sub-clinical chorioamnionitis is a significant primary cause of perinatal deaths.

Placental histology was also pivotal in identifying uteroplacental insufficiency, which helped to confirm the diagnosis of IUGR (Table IV). The diagnosis was changed to IUGR in 30 cases, of which 24 were previously IUDs of unknown aetiology. Chronic villitis, which is usually nonspecific (and termed villitis of unknown aetiology), can be seen in placentas of stillbirths, and has been associated with IUGR. This complication can occur with subsequent pregnancies, depending on the severity of the villitis. ${ }^{10,11}$ Becroft et al., in 509 placentas from SGA infants, found that villitis of unknown aetiology is an independent risk factor for SGA (adjusted odds ratio 2.35, 95\% CI 1.55 - 3.56). ${ }^{12}$ This particular cause of PND presents an opportunity for intervention in the next pregnancy with the availability of antenatal uterine artery and umbilical artery Doppler and the clinical surveillance of patients. $^{7}$

Early screening and treating for syphilis will decrease the PNM rate. The primary cause of death in 18 of our cases was attributed to treponemal infection. This is a high rate for a treatable condition, and it should be routinely screened for. The next most common obstetric cause of death was spontaneous PTL, which has a multi-factorial aetiology. Effective interventions to prevent preterm birth remain elusive. ${ }^{13}$

There is a high incidence of smoking and alcohol abuse in our population, both associated with abruptio placentae and IUGR. ${ }^{14,15} \mathrm{APH}$ is our third most common cause of obstetric death; 24 cases $(60 \%)$ were directly classified as abruptio placentae without hypertension. Patient education and information would help to target this serious obstetric complication that exacerbates PNM and has a considerable impact on maternal morbidity and mortality.

Early antenatal clinic attendance, combined with early ultrasound examination, ensures accurate determination of gestational age and detection of gross fetal anomalies, and eliminates the uncertainty of post-date pregnancies ( 2 cases in the series). Nuchal translucency scans are a viable alternative to biochemical testing for screening for aneuploidy. Patients with fetal anomalies can be offered termination of pregnancy early in the second trimester, which will also tend to reduce PNM.

Because of changed demarcation in 2008 of health districts in Cape Town, resulting in major changes in referral routes, future similar population-based studies and comparisons will not be possible for this geographical area.

\section{Conclusion}

Perinatal care in the Western Cape Province and TBH has improved over the last 2 decades. The TPRL rate has decreased in TBH although the number of deliveries has increased. The addition of a placental histology service has contributed to reducing the proportion of unexplained IUDs. The focus of resources and research should move towards education and preventing infection as the most important cause of TPRL. Identification of risk factors and implementing preventive measures may lead to a further decrease in TPRL.

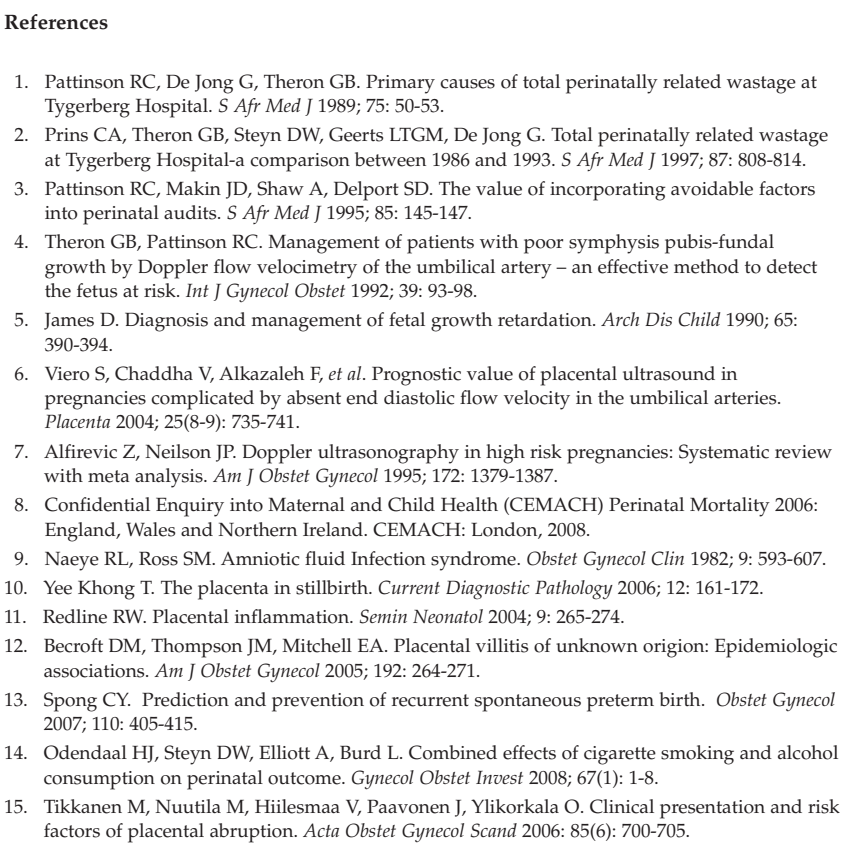

12. Becroft DM, Thompson JM, Mitchell EA. Placental villitis of unknown origion: Epidemiologic associations. Am J Obstet Gynecol 2005; 192: 264-271

13. Spong CY. Prediction and prevention of recurrent spontaneous preterm birth. Obstet Gynecol 2007; 110: 405-415.

14. Odendaal HJ, Steyn DW, Elliott A, Burd L. Combined effects of cigarette smoking and alcohol consumption on perinatal outcome. Gynecol Obstet Invest 2008; 67(1): 1-8.

5. Tikkanen M, Nuutila M, Hiilesmaa V, Paavonen J, Ylikorkala O. Clinical presentation and risk factors of placental abruption. Acta Obstet Gynecol Scand 2006: 85(6): 700-705.

Accepted 6 November 2009. 